\title{
Estudo termoanalítico de comprimidos revestidos contendo captopril através de termogravimetria (TG) e calorimetria exploratória diferencial
} (DSC)

\author{
Giovana Carolina Bazzo, Marcos Antonio Segatto Silva*
}

Centro de Ciências da Saúde, Departamento de Ciências Farmacêuticas, Universidade Federal de Santa Catarina.

* Correspondência:

M. A. S. Silva.

Departamento de Ciências

Farmacêuticas. Universidade Federal

de Santa Catarina. Campus

Universitário, Trindade, 88040-900,

Florianópolis, SC, Brasil.

E-mail: segatto@ccs.ufsc.br
No presente trabalho foram desenvolvidos comprimidos de captopril revestidos com hidroxipropilmetilcelulose (HPMC), Opadry $^{\circledR}$, polivinilpirrolidona (PVP), Eudragit ${ }^{\circledR}$ E e goma laca. Foi realizado estudo termoanalítico do fármaco e das formulações através de termogravimetria (TG) e calorimetria exploratória diferencial (DSC). Através da análise das curvas DSC verificouse que não houve a ocorrência de interação entre o fármaco e os excipientes lactose, celulose microcristalina, croscarmelose sódica, Aerosil $l^{\circledR}$ e talco, utilizados na formulação do comprimido. Através desta técnica detectou-se a possibilidade de interação entre captopril e estearato de magnésio. De acordo com os resultados obtidos através de DSC não foram observadas alterações na cristalinidade do fármaco decorrentes dos processos de compressão e revestimento. A termogravimetria foi utilizada para o estudo da cinética de degradação do captopril e dos comprimidos. Os parâmetros cinéticos foram determinados através do método de Ozawa. Os resultados demonstraram que não houve alteração da estabilidade térmica do captopril na forma de comprimido. A formulação revestida com HPMC foi a que apresentou maior estabilidade térmica, quando comparada às demais formulações de revestimento.

\section{INTRODUÇÃO}

O captopril, 1-[(2S)-3-mercapto-2 metilpropionil]-Lprolina, é um potente inibidor da enzima conversora de angiotensina (ECA) por via oral, sendo indicado no tratamento da hipertensão arterial e insuficiência cardíaca congestiva (Brogden et al., 1988; Garrison, Peach, 1991; Nur, Zhang, 2000). É suscetível de sofrer degradação oxidativa, especialmente quando em solução aquosa (Connors et al., 1986; Taketomo et al., 1990; Martindale, 1993; Trissel, 2000). A velocidade da reação de oxidação depende principalmente do $\mathrm{pH}$, da concentração de oxigênio e da presença de íons metálicos (Connors et al., 1986; Trissel, 2000).

Os polímeros formadores de filme ou film coating surgem atualmente como o processo de escolha para o revestimento de comprimidos. Entre as diversas aplicações do revestimento de formas farmacêuticas sólidas está a proteção do princípio ativo contra agentes externos, principalmente ar, umidade e luz, a fim de aumentar a estabilidade das formulações (Bauer et al., 1998; Porter, 2000; Seitz et al., 2001). 
O estudo da estabilidade é um dos principais fatores que devem ser avaliados no desenvolvimento de formulações. Estes estudos são realizados rotineiramente pela indústria farmacêutica, porém, requerem longos períodos de armazenamento das amostras, sob condições controladas de temperatura e umidade (Ansel et al., 2000; Rodante et al., 2002). As técnicas termoanalíticas apresentam inúmeras vantagens nos estudos de interação entre princípio ativo e excipientes, estudo da cinética de degradação e estabilidade de formas farmacêuticas, entre outras aplicações. Embora não substituam os estudos convencionais, as técnicas termoanalíticas mostramse extremamente úteis em estudos de estabilidade, possibilitando a escolha das formulações mais estáveis com extrema rapidez, fator desejável especialmente para a indústria farmacêutica (Vecchio et al., 2001; Rodante et al., 2002; Souza et al., 2002).

No presente trabalho, realizou-se o estudo termoanalítico do captopril e de formulações de comprimidos revestidos com polímeros (HPMC, Opadry ${ }^{\circledR}$, PVP e Eudragit ${ }^{\circledR}$ E) e também goma laca, uma resina natural. Investigou-se, através da técnica DSC, a possibilidade de ocorrência de interações entre o fármaco e os excipientes lactose, celulose microcristalina, talco, croscarmelose sódica, Aerosil ${ }^{\circledR}$ e estearato de magnésio, os quais foram utilizados na composição dos comprimidos. Também, investigou-se a influência dos processos tecnológicos de compressão e revestimento sobre as propriedades do fármaco. Foi realizado o estudo da cinética de degradação das formulações através de termogravimetria, com o objetivo de verificar a influência dos revestimentos sobre a estabilidade térmica das mesmas.

\section{MATERIAL E MÉTODOS}

\section{Preparo das formulações}

Comprimidos de captopril, contendo $50 \mathrm{mg}$ de fármaco, foram preparados através do método de compressão direta, em compressora LAWES (modelo 200010 PSO), utilizando conjunto de matrizes e punções bicôncavos de $6 \mathrm{~mm}$ de diâmetro. A formulação foi composta por 26,5\% de lactose (Lactose Super - Tab spray dried - Blanver Farmoquímica); 18,0\% de celulose microcristalina (Microcel MC 102 - Blanver Farmoquímica); 0,7\% de croscarmelose sódica (Explocel A - Blanver Farmoquímica); 0,3\% de dióxido de silício coloidal (Aerosil ${ }^{\circledR}$ - Degussa Inc.); $1,5 \%$ de estearato de magnésio e 3,0\% de talco.

$\mathrm{O}$ revestimento dos comprimidos foi realizado em equipamento RAMA COTA modelo R\&D, capacidade 5-
$7 \mathrm{~kg}$, com pistola de aplicação através de sistema de spray com ar e bomba peristáltica. Foram desenvolvidas cinco formulações de revestimento, as quais estão descritas na Tabela I. As condições empregadas no processo de revestimento foram as seguintes: velocidade da drageadeira de 4,5-5,5 rpm, temperatura de $38-40{ }^{\circ} \mathrm{C}$ e vazão da bomba $17-20 \mathrm{~g} / \mathrm{min}$ para as formulações revestidas com HPMC, Opadry ${ }^{\circledR}$ e PVP. Para as revestidas com Eudragit ${ }^{\circledR}$ e goma laca, a velocidade utilizada foi de $8,0-8,5 \mathrm{rpm}$, temperatura de $37-39^{\circ} \mathrm{C}$ e vazão da bomba $15 \mathrm{~g} / \mathrm{min}$.

\section{DSC}

Os excipientes selecionados para o desenvolvimento dos comprimidos, bem como o captopril e misturas 1:1 do fármaco com os adjuvantes, foram submetidos a ensaio termoanalítico de calorimetria exploratória diferencial (DSC), com o objetivo de avaliar possíveis interações entre os componentes da formulação. Também, foram obtidas curvas DSC da mistura física dos componentes antes de submetê-los à compressão, bem como dos comprimidos não revestidos e revestidos.

As curvas DSC foram obtidas através da célula DSC-50 da SHIMADZU, sob as seguintes condições experimentais: atmosfera dinâmica de nitrogênio com vazão de $50 \mathrm{~mL} / \mathrm{min}$; razão de aquecimento de $10{ }^{\circ} \mathrm{C} / \mathrm{min}$, da temperatura ambiente até $500{ }^{\circ} \mathrm{C}$; cápsula de alumínio parcialmente fechada e massa de amostra em torno de 1,5 mg. A célula DSC foi calibrada antes dos ensaios no eixo de temperatura utilizando padrões de índio (Tfusão $=$ $156,6^{\circ} \mathrm{C}$ ) e zinco (Tfusão $=419,5^{\circ} \mathrm{C}$ ) metálicos com pureza de $99,99 \%$. Para a quantidade de calor empregou-se o ' $\mathrm{H}_{\text {fusão }}$ do índio metálico $\left(28,7 \mathrm{Jg}^{-1}\right)$.

\section{TG}

As curvas TG do captopril, dos comprimidos não revestidos e das formulações revestidas foram obtidas através da termobalança TGA-50 da SHIMADZU.

As condições experimentais empregadas foram as seguintes: razões de aquecimento de 2,$5 ; 5,10,20$ e $40{ }^{\circ} \mathrm{C} / \mathrm{min}$, da temperatura ambiente até $650{ }^{\circ} \mathrm{C}$; atmosfera oxidativa (ar comprimido) com vazão de $50 \mathrm{~mL} / \mathrm{min}$; cadinho de platina e massa de amostra em torno de 4,0 mg. Antes dos ensaios verificou-se a calibração do instrumento empregando-se uma amostra de padrão de oxalato de cálcio monoidratado, conforme norma ASTM (1582-93).

Para a determinação dos parâmetros cinéticos, utilizou-se o método de Ozawa (1965), através do software TASYS (SHIMADZU). 
TABELA I - Composição das formulações de revestimento dos comprimidos de captopril

\begin{tabular}{|c|c|c|c|c|c|}
\hline & HPMC (\%) & Opadry $^{\circledR}(\%)$ & PVP (\%) & $\operatorname{Eudragit}^{\circledR}(\%)$ & Goma laca (\%) \\
\hline HPMC(Methocel $\left.^{\circledR}\right)$ & 6,8 & & & & \\
\hline Opadry ${ }^{\circledR}$ Clear & & 6,9 & & & \\
\hline PVP K30 & & & 6,8 & & 0,8 \\
\hline Eudragit ${ }^{\circledR}$ E100 & & & & 10,6 & \\
\hline Goma laca & & & & & 7,5 \\
\hline PEG 400 & 0,7 & & 0,7 & & \\
\hline PEG 6000 & & & & 1,06 & 0,6 \\
\hline Dióxido de titânio & 1,9 & 1,7 & 1,9 & 1,6 & 0,7 \\
\hline CoranteYellow n ${ }^{\circ} 5$ & 0,01 & 0,01 & 0,01 & 0,04 & 0,08 \\
\hline Talco & & & & 5,3 & 2,2 \\
\hline Estearato de magnésio & & & & 2,7 & 1,6 \\
\hline Trietilcitrato & & & & 4,2 & \\
\hline Álcool $96^{\circ} \mathrm{GL}$ & 84,9 & 85,7 & 84,9 & 74,5 & 86,5 \\
\hline Água deionizada & 5,7 & 5,7 & 5,7 & & \\
\hline
\end{tabular}

\section{RESULTADOS}

Na Figura 1 está representada a curva DSC obtida para o captopril.

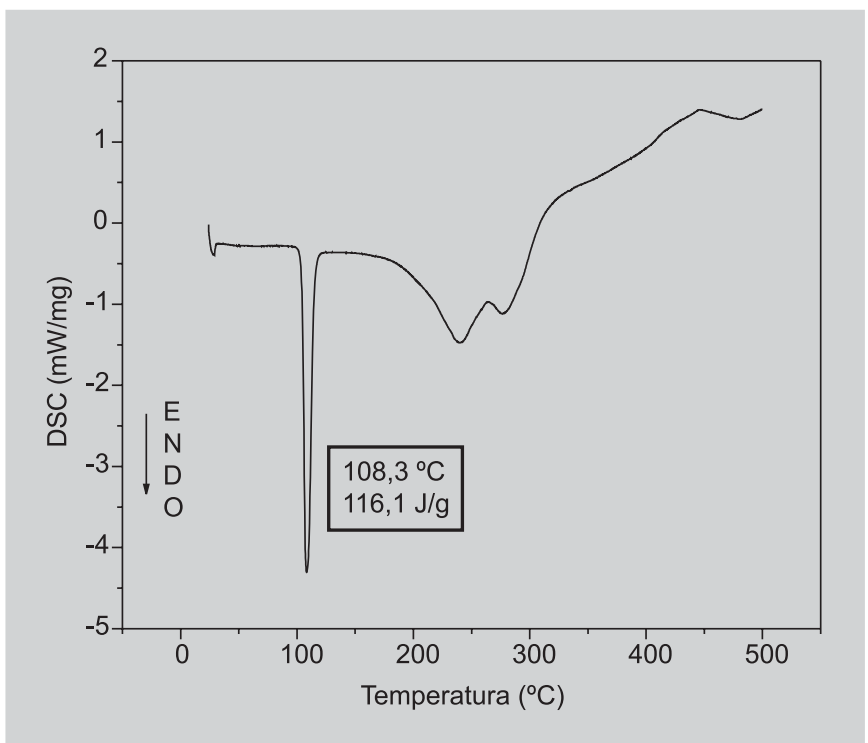

FIGURA 1 - Curva DSC do captopril obtida sob atmosfera dinâmica de nitrogênio e razão de aquecimento de $10^{\circ} \mathrm{C} / \mathrm{min}$.

A Figura 2 corresponde às curvas DSC das misturas 1:1 do fármaco com os excipientes lactose, celulose microcristalina, croscarmelose sódica, Aerosil ${ }^{\circledR}$ e talco. $\mathrm{Na}$ Figura 3 encontram-se as curvas DSC do captopril, estearato de magnésio, bem como da mistura 1:1 dos dois componentes.

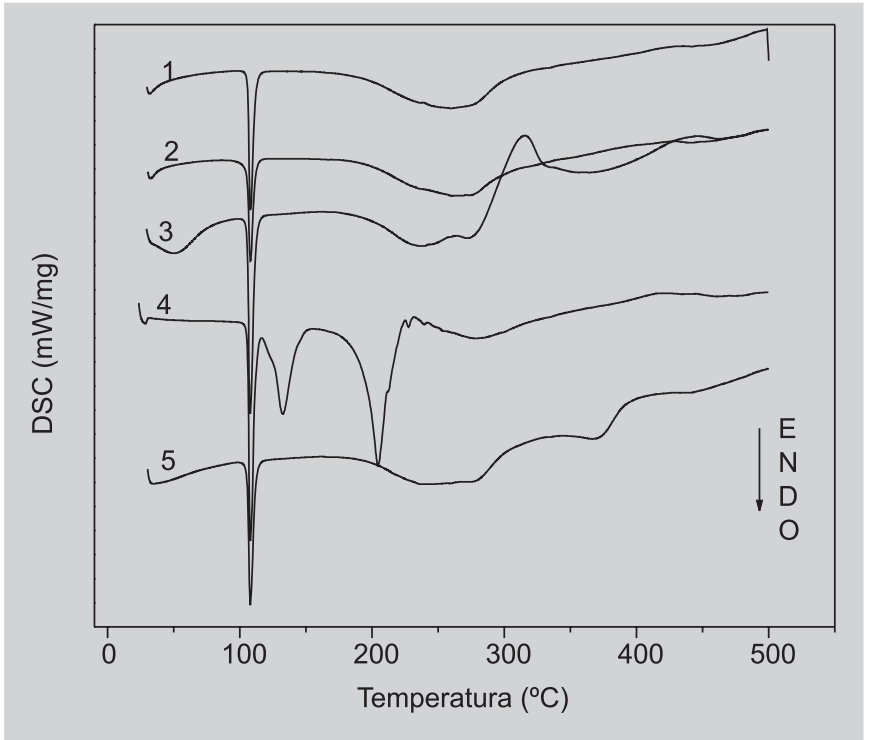

FIGURA 2 - Curvas DSC da mistura 1:1 do captopril com $\operatorname{Aerosil}^{\circledR}(1)$, talco (2), croscarmelose sódica (3), lactose (4) e celulose microcristalina (5) obtidas sob atmosfera dinâmica de nitrogênio e razão de aquecimento de $10^{\circ} \mathrm{C} / \mathrm{min}$.

As curvas DSC da mistura física dos componentes da formulação antes da compressão e dos comprimidos revestidos e não revestidos estão representadas nas Figuras 4 e 5. Na Tabela II estão descritas as temperaturas e entalpias de fusão do captopril, do fármaco associado aos excipientes, bem como das formulações.

As curvas TG obtidas para o captopril e para os comprimidos estão representadas na Figuras 6. No estudo da cinética de degradação das formulações, os 


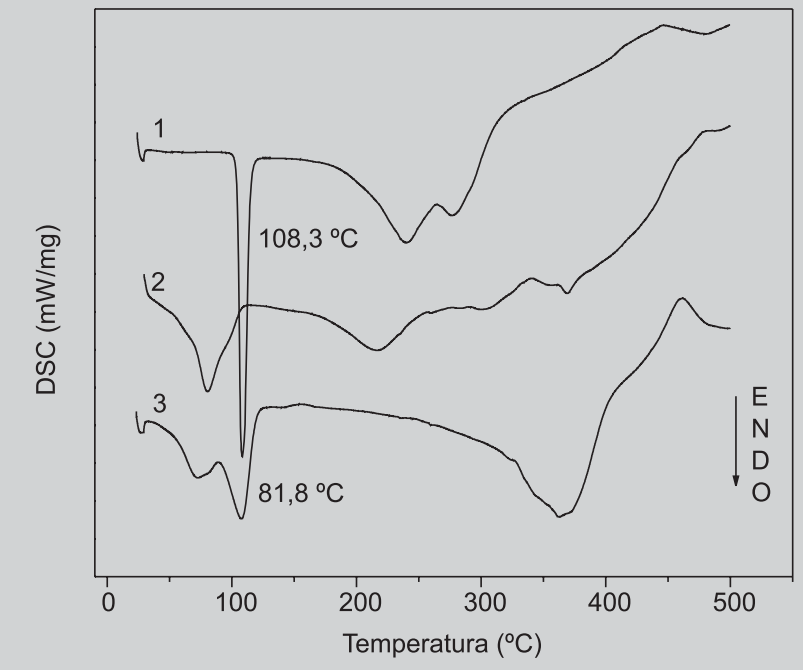

FIGURA 3 - Curvas DSC do captopril (1), estearato de magnésio (2) e mistura 1:1 dos dois componentes (3), obtidas sob atmosfera dinâmica de nitrogênio e razão de aquecimento de $10^{\circ} \mathrm{C} / \mathrm{min}$.

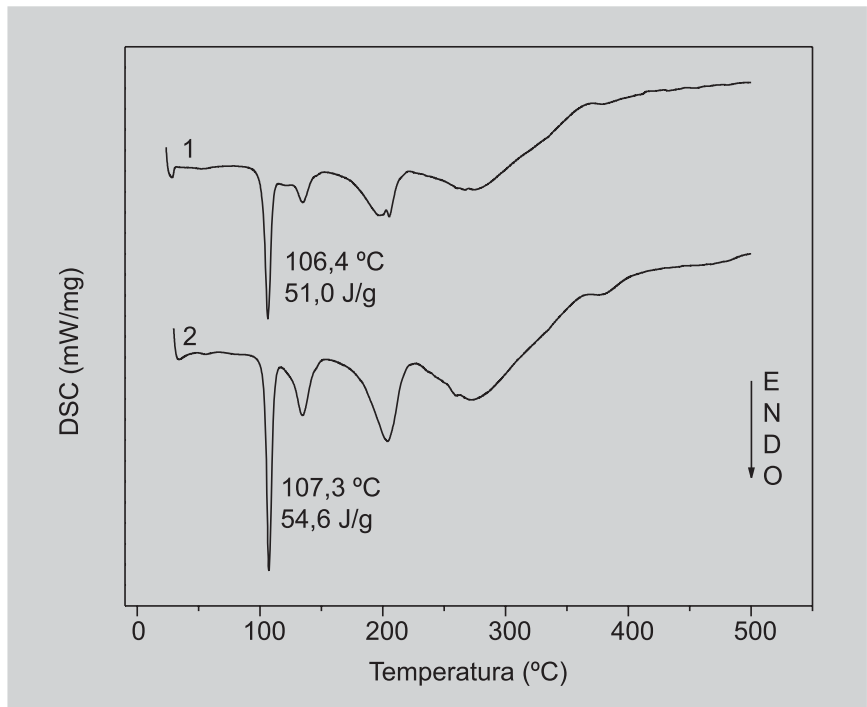

FIGURA 4 - Curvas DSC da mistura física dos componentes da formulação (1) e dos comprimidos não revestidos (2) obtidas sob atmosfera dinâmica de nitrogênio e razão de aquecimento de $10{ }^{\circ} \mathrm{C} / \mathrm{min}$.

parâmetros cinéticos (energia de ativação, ordem de reação e fator freqüência) foram determinados com o auxílio do software TASYS (Shimadzu), através do método de Ozawa e estão descritos na Tabela III.

\section{DISCUSSÃO}

Na curva DSC do captopril (Figura 1) pode-se observar um evento endotérmico a $108,3{ }^{\circ} \mathrm{C}$, correspondente

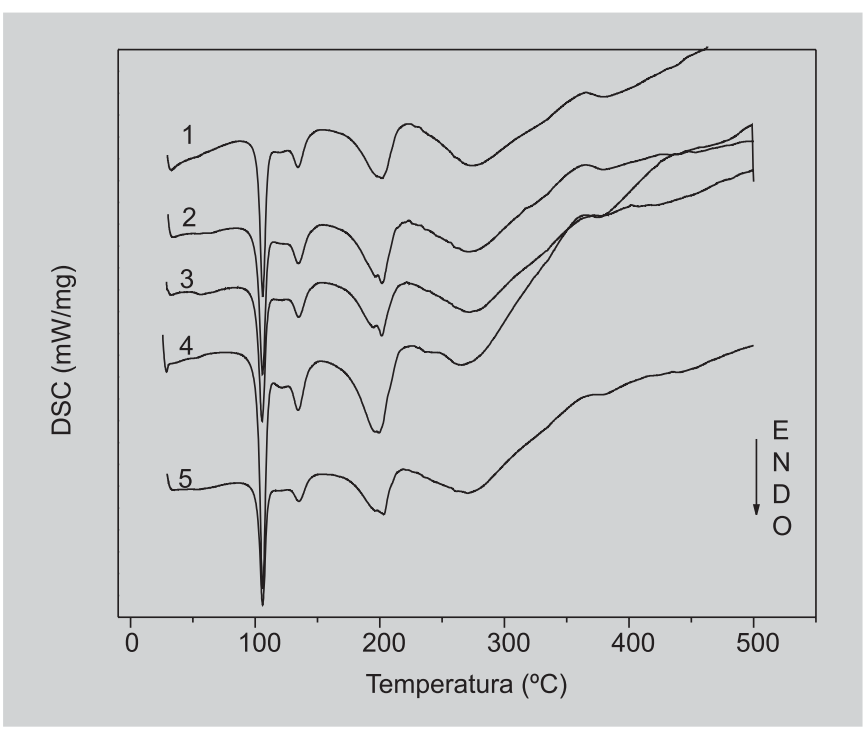

FIGURA 5 - Curvas DSC dos comprimidos revestidos com Opadry $^{\circledR}$ (1), HPMC (2), PVP (3), Eudragit ${ }^{\circledR}$ (4) e goma laca (5), obtidas sob atmosfera dinâmica de nitrogênio e razão de aquecimento de $10^{\circ} \mathrm{C} / \mathrm{min}$.

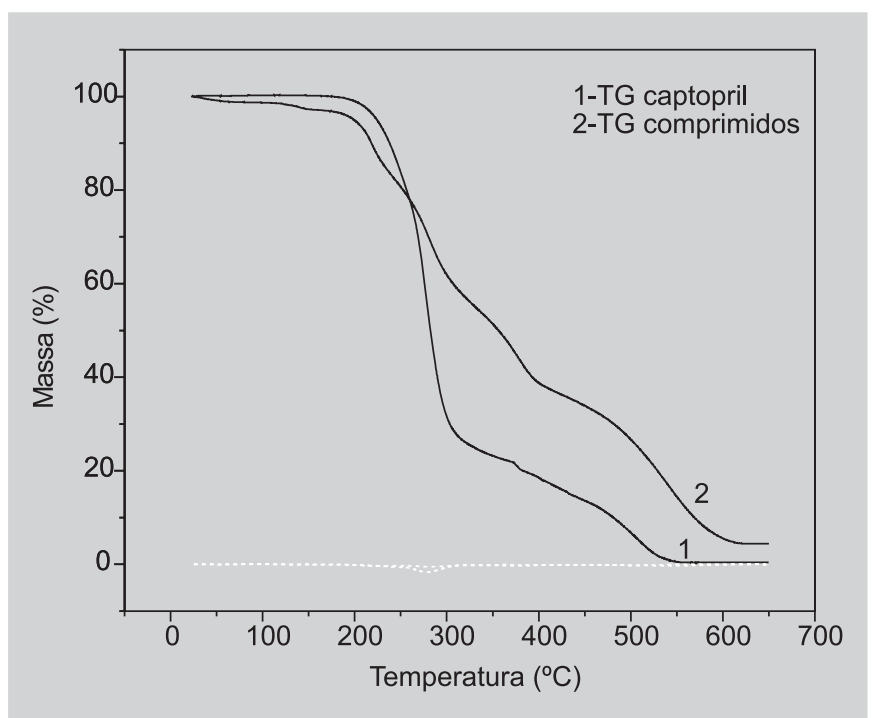

FIGURA 6 - Curvas TG do captopril e dos comprimidos, obtidas sob atmosfera oxidativa (ar comprimido) com vazão de $50 \mathrm{~mL} / \mathrm{min}$ e razão de aquecimento de $10^{\circ} \mathrm{C} / \mathrm{min}$.

à fusão do fármaco, apresentando valor de entalpia de $116,1 \mathrm{~J} \mathrm{~g}^{-1}$. A fusão caracteriza-se por ser um fenômeno físico, o qual pode ser detectado através das curvas DSC ou DTA, apresentando-se como um evento endotérmico (Wendlandt, 1986; Clas et al., 1999). Nas curvas DSC correspondentes à mistura do fármaco com os excipientes (Figura 2) pode-se observar que os eventos térmicos do captopril e dos excipientes citados estão nas mesmas temperaturas em que ocorrem nas curvas DSC das espécies 
TABELA II - Entalpias de fusão do captopril $\left(\mathrm{J} \mathrm{g}^{-1}\right)$ e temperaturas referentes aos eventos $\left({ }^{\circ} \mathrm{C}\right)$

\begin{tabular}{|c|c|c|}
\hline & ${ }^{\prime} \mathrm{H}\left(\mathrm{J} \mathrm{g}^{-1}\right)$ & Temperatura $\left({ }^{\circ} \mathrm{C}\right)$ \\
\hline Captopril & 116,1 & 108,3 \\
\hline Mistura física & 54,6 & 107,3 \\
\hline Comprimidos & 51,0 & 106,4 \\
\hline Comprimidos revestidos (HPMC) & 47,3 & 105,9 \\
\hline Comprimidos revestidos $\left(\right.$ Opadry $\left.{ }^{f}\right)$ & 46,3 & 106,0 \\
\hline Comprimidos revestidos (PVP) & 46,8 & 105,9 \\
\hline Comprimidos revestidos $\left(\right.$ Eudragit $\left.^{\ddagger}\right)$ & 44,9 & 105,8 \\
\hline Comprimidos revestidos (Goma laca) & 48,9 & 105,82 \\
\hline
\end{tabular}

TABELA III - Parâmetros cinéticos energia de ativação (Ea), ordem de reação (n) e fator freqüência (A) obtidos para o captopril e para as formulações através do método de Ozawa

\begin{tabular}{lccc}
\hline & $\mathrm{Ea}(\mathrm{kJ} / \mathrm{mol})$ & $\mathrm{n}$ & $\mathrm{A}\left(\mathrm{min}^{-1}\right)$ \\
\hline Captopril & 110,8 & 5,0 & $1,560.10^{10}$ \\
Comprimidos & 110,2 & 4,8 & $3,241.10^{10}$ \\
Comprimidos revestidos (HPMC) & 111,9 & 4,2 & $4,511.10^{10}$ \\
Comprimidos revestidos (Opadry ${ }^{\mp}$ ) & 103,4 & 4,4 & $5,536.10^{9}$ \\
Comprimidos revestidos (PVP) & 103,7 & 3,8 & $5,748.10^{9}$ \\
Comprimidos revestidos (Eudragit $^{\mp}$ ) & 106,2 & 5,0 & $1,224.10^{9}$ \\
Comprimidos revestidos (Goma laca) & 103,4 & 3,7 & $5,703.10^{9}$ \\
\hline
\end{tabular}

puras. Nenhum evento adicional foi observado, sugerindo a não ocorrência de interação entre o fármaco e estes adjuvantes.

Entretanto, na curva DSC, que corresponde à mistura entre captopril e estearato de magnésio (Figura 3), houve diminuição da temperatura de fusão do captopril para $81,82{ }^{\circ} \mathrm{C}$, bem como alterações na forma do pico. Este fato pode sugerir a presença de interação entre os dois componentes, visto que a primeira endoterma é devida à desidratação do estearato de magnésio e a segunda evidencia o abaixamento no ponto de fusão do fármaco. Observação semelhante foi feita durante os estudos de interação entre picotamida e ácido esteárico (Mura et al., 1998b).

Conforme pode-se observar na Tabela II, houve alteração no valor da entalpia de fusão do captopril de $116,1 \mathrm{~J} \mathrm{~g}^{-1}$ para 54,6 $\mathrm{J} \mathrm{g}^{-1}$ quando associado aos excipientes (mistura física). Este valor equivale a praticamente a metade do valor de ' $\mathrm{H}$ do captopril puro, visto que se trata de misturas físicas na proporção 1:1. A fusão do fármaco nessas misturas ocorreu de forma inalterada, apenas apresentando um pequeno abaixamento. No entanto, pode-se observar que os eventos térmicos mais significativos ocorrem nas mesmas faixas de temperatura, com pequenas alterações (Figura 4). Estas pequenas alterações estão relacionadas à presença dos excipientes na formulação, porém, não caracterizam a ocorrência de interações (Mura et al., 1998a; Macedo, Nascimento, 2001).

Comparando-se a curva DSC da mistura física dos componentes da formulação com a curva DSC após compressão (comprimidos), pode-se observar que não houve alterações significativas que sugerissem modificações nas propriedades do fármaco decorrentes do processo de compressão. Da mesma forma, não foram observadas alterações nas curvas DSC dos comprimidos revestidos após o processo tecnológico de revestimento (Figuras $4 \mathrm{e}$ 5). Todos os eventos térmicos característicos permaneceram presentes e não foram observadas alterações na forma e área do pico correspondente à fusão do captopril. Modificações quanto à cristalinidade dos fármacos podem ser caracterizadas por DSC através de alterações significativas nas temperaturas e valores de entalpia referentes aos eventos, alterações na área e forma dos picos, bem como pelo aparecimento de novos eventos. Estas observações podem sugerir a ocorrência de alterações na cristalinidade, porém devem ser comprovadas com o auxílio de técnicas adicionais, a exemplo de difratometria de raios-X ou espectroscopia de absorção na região do 
infravermelho (Lin et al., 1994; Mura et al., 1995; Matsunaga et al., 1996; Mura et al., 1998a; Mura et al., 1998b).

Huang e colaboradores (2001) realizaram estudo termoanalítico do captopril e verificaram que o fármaco sofreu decomposição térmica entre 160 e $450{ }^{\circ} \mathrm{C}$, utilizando atmosfera dinâmica de nitrogênio com vazão de $100 \mathrm{~mL} / \mathrm{min}$. Conforme pode-se observar na Figura 6, o captopril decompôs-se na faixa de temperatura de 160 a $550{ }^{\circ} \mathrm{C}$ utilizando-se as condições descritas neste trabalho. De acordo com as curvas TG dos comprimidos pode-se observar que o processo de decomposição térmica iniciase na mesma temperatura que o captopril puro (aproximadamente $160^{\circ} \mathrm{C}$ ), evidenciando que os excipientes não influenciam a decomposição térmica do fármaco.

A energia de ativação é um dos parâmetros de maior interesse para avaliar a estabilidade de compostos farmacêuticos (Vecchio et al., 2001). De acordo com Macedo et al. (2000), pode-se estabelecer um perfil comparativo de estabilidade entre vários fármacos através dos valores obtidos de $E a$, em que quanto maior a energia de ativação, maior a estabilidade térmica do composto.

No estudo da cinética de degradação das formulações contendo captopril, pode-se observar através dos valores de energia de ativação (Tabela III) que o princípio ativo puro apresenta praticamente a mesma estabilidade térmica do que na presença de excipientes. Os valores obtidos foram muito próximos, sendo de $110,8 \mathrm{~kJ} / \mathrm{mol}$ para o fármaco e $110,2 \mathrm{~kJ} / \mathrm{mol}$ para a forma de comprimido.

Os comprimidos não revestidos e os comprimidos revestidos também apresentaram valores de energia de ativação bastante próximos, sugerindo que todas as formulações apresentam praticamente a mesma estabilidade térmica. No entanto, foi possível traçar um perfil comparativo. A formulação revestida com HPMC foi a que apresentou maior energia de ativação $(111,9 \mathrm{~kJ} / \mathrm{mol})$, sendo considerada a mais estável, seguida dos comprimidos sem revestimento $(110,2 \mathrm{~kJ} / \mathrm{mol})$. A revestida com Eudragit ${ }^{\mathbb{B}}$ apresentou energia de ativação de $106,2 \mathrm{~kJ} / \mathrm{mol}$, possuindo estabilidade térmica intermediária, quando comparada às demais formulações. Por fim, as formulações revestidas com PVP, Opadry ${ }^{\circledR}$ e goma laca foram as que apresentaram menor estabilidade térmica, apresentando valores de energia de ativação de 103,$8 ; 103,4$ e $103,4 \mathrm{~kJ} / \mathrm{mol}$, respectivamente.

\section{CONCLUSÕES}

Os resultados obtidos através de DSC não evidenciaram a ocorrência de interações entre o captopril e celulose microcristalina, lactose, croscarmelose sódica, tal- co e Aerosil ${ }^{\circledR}$. Os resultados sugerem que estes excipientes podem ser utilizados em formulações contendo o fármaco. No entanto, pode-se sugerir a possibilidade de ocorrência de interação entre o captopril e o estearato de magnésio.

Não foram observadas modificações na cristalinidade do fármaco decorrentes dos processos tecnológicos de compressão e revestimento, utilizando a técnica DSC.

De acordo com os resultados obtidos no estudo da cinética de degradação, as formulações de revestimento desenvolvidas, aplicadas nas condições estabelecidas, não evidenciaram aumento significativo da estabilidade térmica do captopril.

\section{ABSTRACT}

\section{Thermal analysis study of captopril coated tablets by thermogravimetry (TG) and differential scanning calorimetry (DSC)}

In the present study, captopril coated tablets with hydroxypropylmethylcellulose (HPMC), Opadry ${ }^{\circledR}$, polyvinylpirrolidone (PVP), Eudragit ${ }^{\mathbb{\circledR}}$ and shellac were produced. Differential scanning calorimetry (DSC) and thermogravimetry (TG) were used to evaluate the thermal properties of the drug and the formulations. On the basis of DSC results, captopril was found to be compatible with lactose, microcrystalline cellulose, sodium croscarmellose, Aerosil ${ }^{\mathbb{B}}$ and talc. Some possibility of interaction between drug-excipient was observed with magnesium stearate. However, additional techniques to confirm the results obtained are needed. There was no influence of mechanical treatment (tableting and coating) on drug crystallinity, in compliance with the DSC results. Thermogravimetry was used to determine the thermal parameters for the captopril drug and its tablets. The kinetic parameters were determined via Ozawa model. The captopril drug and tablet presented the same thermal stability. The tablets coated with HPMC presented the highest thermal stability.

UNITERMS: Captopril. Coated tablets. Thermogravimetry (TG). Differential scanning calorimetry (DSC). Interaction. Stability.

\section{AGRADECIMENTOS}

Os autores agradecem a Blanver Farmoquímica Ltda. e o Laboratório de Análise Térmica Prof. Ivo Giolito, pelo apoio concedido para realização deste trabalho. 


\section{REFERÊNCIAS BIBLIOGRÁFICAS}

ANSEL, H. C.; POPOVICH, N. G.; ALLEN JR., L. V. Formas Farmacêuticas e Sistemas de Liberação de Fármacos. São Paulo: Editorial Premier, 2000. p. 113140,201-235.

BAUER, K. H.; LEHMANN, K.; OSTERWALD, H . P. ROTHGANG, G. Coated pharmaceutical dosage forms. Sttutgart: Medpharm Scientific Publishers, 1998. $280 \mathrm{p}$.

BROGDEN, R. N.; TODD, P. A.; SORKIN, E. M. Captopril: an update of its pharmacodinamic and pharmacokinetic properties, and therapeutic use in hypertension and congestive heart failure. Drugs, v.36, n.5, p. 542-587, 1988.

CLAS, S. D.; DALTON, C. R.; HANCOCK, B. C. Differential scanning calorimetry: applications in drug development. PSTT, v.2, n.8, p. 311-320, 1999.

CONNORS, K. A.; AMIDON, G. L.; STELLA, V. J. Chemical Stability of Pharmaceuticals. 2 ed. New York: John Wiley \& Sons, 1986. p. 284-289.

HUANG, Y.; CHENG, Y.; ALEXANDER, K.; DOLLIMORE, D. The thermal analysis study of the drug captopril. Thermochim. Acta, v. 367-368, p. 43-58, 2001.

GARRISON, J. C.; PEACH, M. J. Tratamento da insuficiência cardíaca congestiva In: As bases farmacológicas da terapêutica. 8 ed. Rio de Janeiro: Guanabara Koogan, 1991. p. 582-592.

LIN, S. H.; CHENG, C. L.; PERNG, R. I. Solid state interaction studies of drug - polymers (II): warfarin Eudragit E, RL or S resins. Eur. J. Pharm. Sci., v. 1, p. 313-322, 1994.

MACEDO, R. O.; NASCIMENTO, T. G.; ARAGÃO, C. F. S.; GOMES, A. P. B. Application of thermal analysis in the characterization of anti-hypertensive drugs. J. Therm. Anal. Calorim., v.59, n.3, p. 657-661, 2000.

MACEDO, R. O.; NASCIMENTO, T. G. Thermal characterization of lapachol by means of TG and DSC coupled to a photovisual system. J. Therm. Anal. Calorim., v. 64, p. 751-756, 2001.
MARTINDALE - The Extra Pharmacopoeia. 30.ed. London: Pharmaceutical Press, 1993. 2363 p.

MATSUNAGA, Y.; BANDO, N.; YUASA, H.; KANAYA, Y. Effects of grinding and tableting on physicochemical stability of an anticancer drug, TAT-59. Chem. Pharm. Bull., v. 44, n. 10, p. 1931-1934, 1996.

MURA, P.; MURA, P.; MANDERIOLI, A.; BRAMANTI, G.; FURLANETTO, S.; PINZAUTI, S. Utilization of differential scanning calorimetry as a screening technique to determine the compatibility of ketoprofen with excipients. Int. J. Pharm., v.119, p. 71-79, 1995.

MURA, P.; MURA, P.; FAUCCI, M. T.; MANDERIOLI, A.; BRAMANTI, G.; CECCARELLI, L. Compatibility study between ibuproxam and pharmaceutical excipients using differential scanning calorimetry, hot-stage microscopy and scanning electron microscopy. J. Pharm. Biomed. Anal., v. 18, p. 151-163, 1998 a.

MURA, P.; FAUCCI, M. T.; MANDERIOLI, A.; FURLANETTO, S.; PINZAUTI, S. Thermal analysis as a screening technique in preformulation studies of picotamide solid dosage forms. Drug Dev. Ind. Pharm., v. 24, n. 8 , p. $747-756,1998$ b.

NUR, A. O.; ZHANG, J. S. Recent progress in sustained/ controlled oral delivery of captopril: an overview. Int. J. Pharm., v. 194, p. 139-146, 2000.

OZAWA, T. A new method of analyzing thermogravimetric data. Bull. Chem. Soc. Jpn., v. 38, n. 11, p. 1881-1886, 1965.

PORTER, S. C. In: Remington: the science and practice of pharmacy. 20.ed. Baltimore: Lippincott Williams \& Wilkins, 2000.2077 p.

RODANTE, F.; VECCHIO, S.; CATALANI, G.; TOMASSETTI, M. Compatibility between active components of a commercial drug. Farmaco, v. 57, p. 833-843, 2002.

SEITZ, J. A.; MEHTA, S. P.; YEAGER, J. L. Teoria e prática na indústria farmacêutica. Lisboa: Fundação Calouste Gulbenkian, 2001. v. 2, p. 599-649.

SOUZA, F. S.; MACEDO, R. O.; VERAS, J. W. E. Studies of cimetidine pre-formulated and tablets for TG and DSC coupled to the photovisual system. Thermochim. Acta, v. 392-393, p. 99-106, 2002. 
TAKETOMO, C. K., CHU, S. A.; CHENG, M. H.; CORPUZ, R. P. Stability of captopril in powder papers under three storage conditions. Am. J. Hosp. Pharm., v. 47, p. 1799-1781, 1990.

TRISSEL, L. A. Trissel's stability of compounded formulations. 2.ed. Washington: American Pharmaceutical Association, 2000. 444p.
VECCHIO, S.; RODANTE, F.; TOMASSETTI, M. Thermal stability of disodium and calcium phosphomycin and the effects of the excipients evaluated by thermal analysis. J. Pharm. Biomed. Anal., v. 24, p. 1111-1123, 2001.

WENDLANDT, W. W. Thermal analysis. 3 ed. New York: John Wiley \& Sons, 1986. 814 p.

Recebido para publicação em 24 de junho de 2003. Aceito para publicação em 01 de setembro de 2005. 